\title{
Rhamnus velutina Boiss. subsp. velutina y Cyperus glaber L., novedosos para la flora de la provincia de Córdoba (España)
}

\section{Jesús Miguel Muñoz Álvarez', María Luisa Díaz² \& Juan Antonio Devesa1}

'Departamento de Botánica, Ecología y Fisiología Vegetal. Edificio José Celestino Mutis, Campus de Rabanales, Universidad de Córdoba, 14071 (Córdoba).

${ }^{2} \mathrm{C} /$ Donantes de Sangre $\mathrm{n}^{\circ}$ 32, 14012 (Córdoba).

\section{Correspondencia}

J.M. Muñoz Álvarez

e-mail: bv1mualj@uco.es

Recibido: 13 septiembre 2017

Aceptado: 24 noviembre 2017

Publicado on-line: diciembre 2017
Rhamnus velutina Boiss. subsp. velutina and Cyperus glaber $L$. new for the Flora of Cordoba province (Spain)

Palabras clave: flora vascular, corología, Córdoba.

Key words: vascular flora, chorology, Córdoba.
Rhamnus velutina Boiss., Elench. PI. Nov: 29 (1838)

CÓRDOBA. Priego de Córdoba, Zamoranos, Cerro de Los Collados, 30 S UG9653, 518 m.s.n.m., 23-V-2017, J. A. Devesa \& J. M. Muñoz (COFC 64568); ibídem, 14VI-2017, J. M. Muñoz (COFC 64567).

Especie nativa del noroeste de África (Marruecos, y Túnez) y de Andalucía: provincias de Cádiz, Sevilla (Muñoz, 1987), Granada, Málaga y Almería (Rivas Martínez \& Pizarro, 2015).

Se ha encontrado en Córdoba, en el cerro de los Collados, ubicado en la zona de transición entre las Sierras Subbéticas y la depresión del Guadalquivir. La población se localiza en las partes altas del mismo, por encima de los 500 m.s.n.m., en calizas y dolomías triásicas embebidas en el seno de los materiales blandos (arcillas y margas del Mioceno) que conforman esta colina, dedicada al cultivo de olivo. La mayor parte de los individuos se encuentran formando un rodal de matorral alto, en un fragmento de espinar, junto con Asparagus acutifolius L., A. albus L., Olea europea L. var. sylvestris (Mill.) Lehr, Osyris alba L., etc., y de manera puntual, pero también en afloramientos calcáreos, se encontraron individuos aislados (aunque frecuentes), en pseudoestepas de Brachypodium retusum (Pers.) P. Beauv., espartales de Macrochloa tenacissima (L.) Kunth y tomillares (de Saturejo micranthae-Thymbrion capitatae Rivas Goday \& Rivas-Martínez), e incluso como rupícola.

El hallazgo de esta especie amplía a la provincia de Córdoba su presencia en Andalucía Occidental.
Cyperus glaber L, Mant. PI. 2: 179 (1771)

- C. bellus sensu Castrov. in Fl. Iber. 18: 15 (2007), non Kunth, Enum. PI. 2: 52 (1837)

CÓRDOBA. Montoro, arroyo de Martín Gonzalo, 30SUH8112, 180 m.s.n.m., 31-08-2012, M. L. Díaz \& J. M. Muñoz (COFC 64569). Baena, Laguna de Casasola, 30SUG8780, 288 m.s.n.m., 1-08-2014, M. L. Díaz \& J. M. Muñoz (COFC 64570).

Especie descrita de Italia ("Habitat in Veronae humentibus") pero cuya área de distribución se extiende por el centro y sur de Europa, algunas islas del Mediterráneo, suroeste y sur de Asia, alcanzando por el este Pakistán (Verloove \& Mesterházy, 2013). Se trata de un taxón controvertido, de distribución todavía mal conocida, cuya presencia en la Península Ibérica se restringía a una copiosa población de los bordes de la Laguna Chica, en las inmediaciones de Moral de Calatrava (Ciudad Real), el límite hasta ahora más occidental de su área de distribución (Castroviejo, 2005). Sin embargo, estas plantas fueron inicialmente identificadas por Castroviejo (2005) como C. rubicundus Vahl y, más tarde, como $C$. bellus Kunth en su tratamiento taxonómico para Flora iberica (G.C. Tucker sec. Castroviejo, 2007), identificaciones discutidas y cuestionadas por Verloove \& Sánchez Gullón (2010), hasta que recientemente se ha aclarado la verdadera identidad de las plantas españolas (Verloove \& Mesterházy, 2013).

Se han localizado dos nuevas poblaciones de esta rara especie, ambas en la provincia de Córdoba y distantes entre sí unos $33 \mathrm{~km}$. La primera se ubica en un tramo del curso bajo del 
arroyo Martín Gonzalo, de agua dulce y neutrófila, en terrenos agrícolas dedicados al olivar. La población se asienta sobre suelos ligeramente arenosos y nitrificados, removidos, húmedos, junto a los pequeños brazos en que se desdobla el arroyo durante el estío, flanqueados por tamujo [Fluggea tinctoria (L.) G.L. Webster]. Se trata de una comunidad de terófitos -dominantes- y hemicriptófitos, de baja altura $(10-12 \mathrm{~cm})$ y escasa cobertura (50\%), que ocupa una pequeña extensión junto a los cursos de agua, pobre en especies (11 taxones). En ella conviven especies propias de comunidades terofíticas estivo-otoñales de IsoetoNanojuncetea Br.-Bl. \& Tüxen ex Westhoff, Dijk \& Passchier [como Cyperus flavescens L., Fimbristylis bisumbellata (Forssk.) Bubani, Gnaphalium luteoalbum L.], de prados antropizados de MolinioArrhenatheretea Tüxen (Cyperus eragrostis Lam., Mentha suaveolens Ehrh., etc.), de malas hierbas de Stellarietea mediae Tüxen, Lohmeyer \& Preising \& ex von Rochow [Xanthium orientale subsp. italicum (Moretti) Greuter y Paspalum distichum L.], etc. Hacia las zonas más húmedas y arcillosas, la población estudiada entra en contacto catenal con una comunidad de Ludwigia palustris (L.) Elliott y Cyperus fuscus L. Por otro lado, la población hallada en los bordes y periferia de la laguna de Casasola (laguna estacional con aguas subsalinas-hiposalinas; Moreira \& Montes, 2005), se asienta sobre materiales salinos y yesíferos del Triásico. Aquí, los individuos de C. glaber forman parte de una comunidad de Verbenion supinae Slavnic (Isoeto-Nanojuncetea), integrada por Crypsis aculeata (L.) Aiton, Heliotropium supinum L., Verbena supina L., Damasonium polyspermum Coss., D. bourgaei Coss., Lythrum tribracteatum Spreng., y $L$. acutangulum Lag., entre otras.

Respecto del estatus de residencia de $C$. glaber en España, Verloove \& Mesterházy (2013) se decantan por considerarla como especie no nativa de España, no existiendo certeza alguna sobre si se trata de una introducción natural o propiciada por el hombre. Las plantas cordobesas se ajustan fielmente a los caracteres diagnósticos señalados por Castroviejo (2007, sub. C. bellus) y Verloove \& Mesterházy (2013), si bien muestran algunas espiguillas que llegan a alcanzar $16 \mathrm{~mm}$ de longitud, y hasta 26 flores, rango que amplía la variabilidad indicada por estos autores.

Se trata de un hallazgo novedoso para la flora de Córdoba y Andalucía, y la segunda cita del taxón para la flora de la Península Ibérica.

\section{Referencias}

Castroviejo, S. (2005). Notas sobre algunos Cyperus alóctonos en Flora iberica. Acta Bot. Malacitana, 31, 241-244.

Castroviejo, S. (2007). Cyperus. In: S. Castroviejo et al. (eds.) Flora iberica,18, 8-27. CSIC. Madrid.

Moreira, J.M. \& C. Montes (coords.) (2005). Caracterización ambiental de humedales en Andalucía. Consejería de Medio Ambiente. Junta de Andalucía.

Muñoz, J. M. (1987). Rhamnus. In: B. Valdés, S. Talavera \& E.F. Galiano (eds.) Flora Vascular de Andalucía Occidental, 2,242-245. Ed. Ketres. Barcelona.

Rivas Martínez, S. \& J. M. Pizarro (2015). Rhamnus. In: F. Muñoz et al. (eds.) Flora iberica, 9, 11-50. CSIC. Madrid.

Verloove, F. \& E. Sánchez Gullón (2010). Further notes on Cyperaceae in the Iberian Peninsula: corrections, adjustments and additions. Fl. Medit., 20, 141-147.

Verloove, F. \& A. Mesterházy (2013). Cyperus glaber L. (Cyperaceae), an enigmatic species "new" to Spain. Webbia, 68(1), 67-71. 